
Pesquisa

DEI

DEPARTAMENTO

DE ENGENHARIA

INDUSTRIAL.

Número 07 | 052021

\title{
Seleção de cenários com Weighted Set
}

\section{Covering}

\author{
Autor(es): \\ Isabella Fischer Guindani Vieira
}




\title{
Série dos Seminários de Acompanhamento à Pesquisa
}

\section{Seleção de cenários com Weighted Set Covering}

\author{
Autor(es): \\ Isabella Fischer Guindani Vieira
}

CRÉDITOS:

SISTEMA MAXWELL / LAMBDA

https://www.maxwell.vrac.puc-rio.br/

Organizadores: Fernanda Baião / Soraida Aguilar 


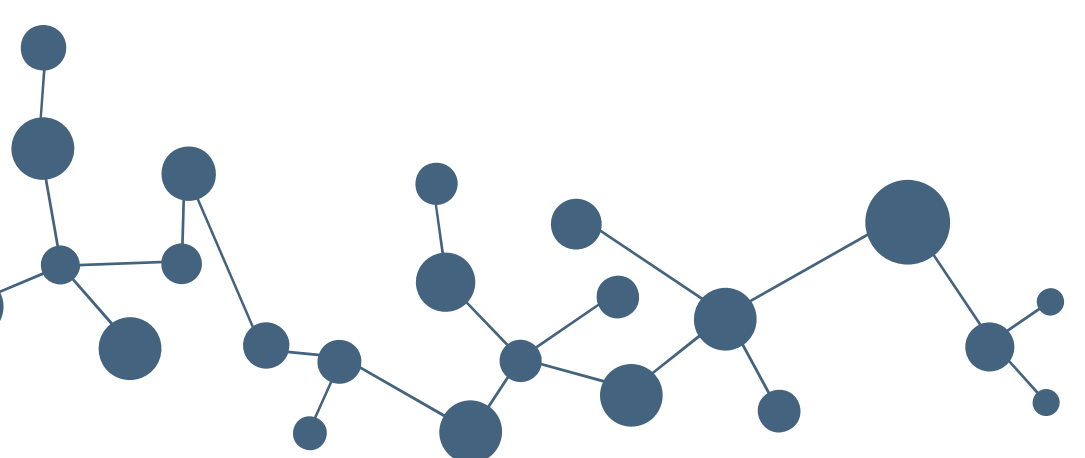

\section{Isabella Fischer}

Mestrado

Áres de concentração: Transporte e Logística

Linha de Pesquisa: Planejamento de Sistemas de Transporte e Logística

$5^{\circ}$ período

Orientador: Rafael Martinelli

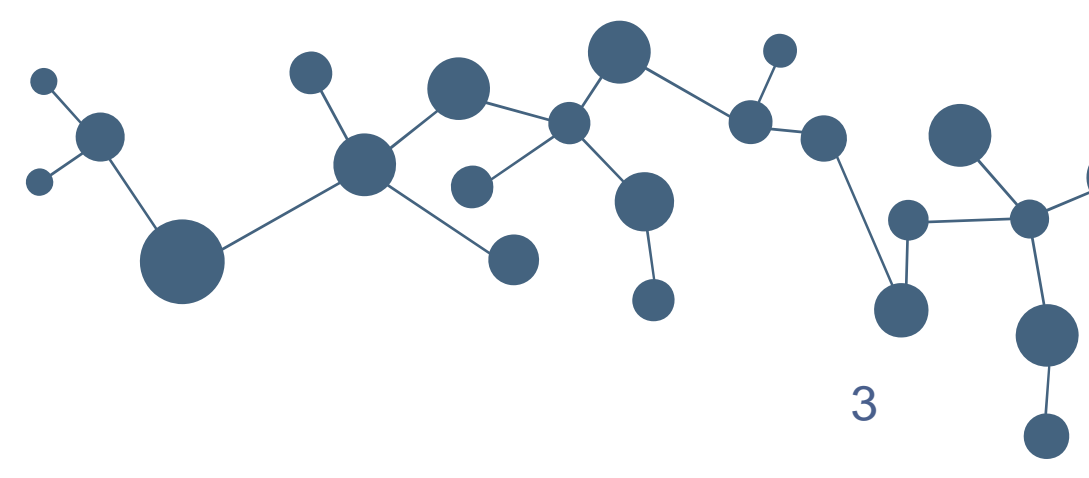




\section{Observação e identificação do problema}

- Contexto geral: cálculo de Demanda Estocástica de ferramentas e materiais em empresa de grande porte do setor de energia.

- Planejamento determinístico com um horizonte extenso, grande número de tarefas e suscetível a diferentes fontes de incerteza. 


\section{Observação e identificação do problema}

Incertezas são calculadas e definidas a partir de dados históricos de cada tarefa e refletem em mudanças nos atributos:

- Data de início;

- Duração;

- Continuidade da sequência de tarefas. 


\section{Observação e identificação do problema}

- Devido à confidencialidade dos dados históricos, as distribuições de probabilidade destas incertezas não são disponibilizadas a todos os setores, incluindo o setor responsável pelo cálculo de demanda de equipamentos.

- Para garantir a correta representação das distribuições, são utilizadas milhares de replicações do cronograma determinístico, aplicando valores sorteados na distribuição de cada tipo de incerteza, para cada atributo. 


\section{Observação e identificação do problema}

CRONOGRAMA

DETERMINÍSTICO

CRONOGRAMA 1

CRONOGRAMA 2

CRONOGRAMA 3

\begin{abstract}
A
\end{abstract}

\begin{tabular}{|l|l|}
\hline A & B \\
\hline
\end{tabular}

A

\begin{tabular}{|c|}
\hline B \\
\hline C \\
\hline B \\
\hline
\end{tabular}

D

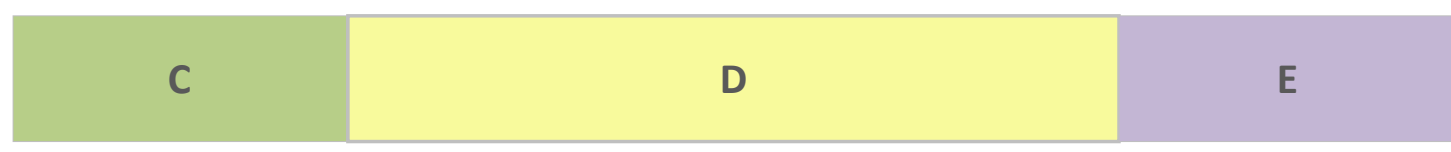

D

E

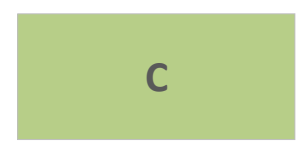

E

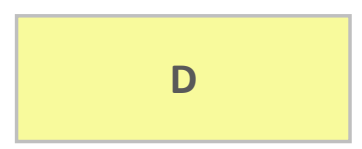




\section{Observação e identificação do problema}

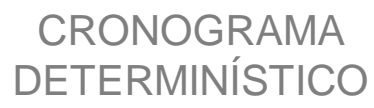

CRONOGRAMA 1

CRONOGRAMA 2

CRONOGRAMA 3

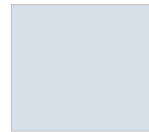

A

\begin{tabular}{|l|l|}
\hline A & B \\
\hline
\end{tabular}

A

\begin{tabular}{|c|}
\hline B \\
\hline B \\
\hline
\end{tabular}

C c

D

E

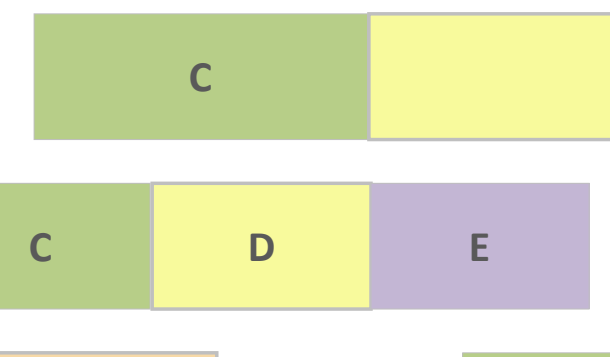

A

C

D

\section{$\vdots$}

\section{C}

D

E 


\section{Observação e identificação do problema}

CRONOGRAMA
DETERMINÍSTICO

CRONOGRAMA 1

CRONOGRAMA 2

CRONOGRAMA 3

CRONOGRAMA 78

A

B

C

D

E

\begin{tabular}{ll} 
A & B \\
\hline
\end{tabular}

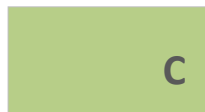

C

D

E

A

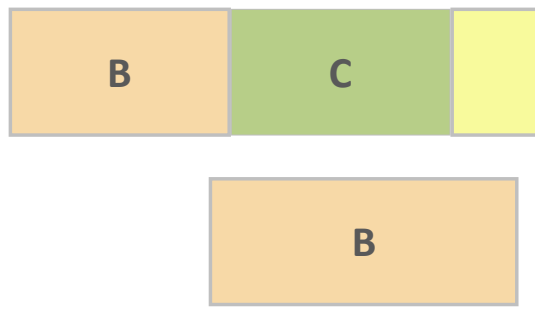

E

A

c

D

A

A

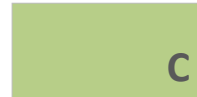

D

E 


\section{Observação e identificação do problema}

- Estes cenários semelhantes causam impacto na demanda que justifique todo este custo de tempo e processamento de dados?

- Para responder tal pergunta foi realizada uma análise de sensibilidade da demanda calculada ao variar o número de replicações:

$$
3000-1000-500-200-100-50
$$




\section{Observação e identificação do problema}

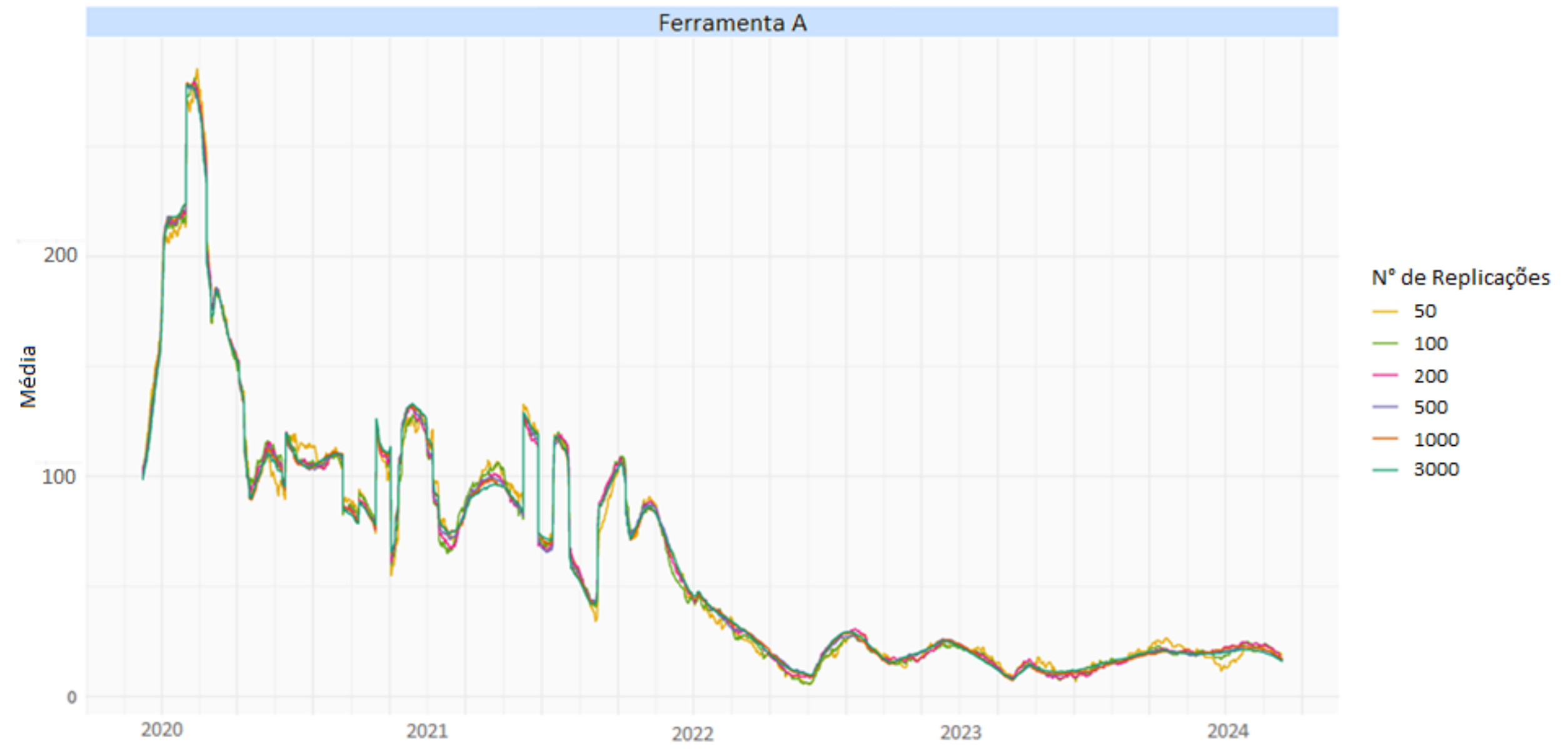




\section{Observação e identificação do problema}

Ferramenta A

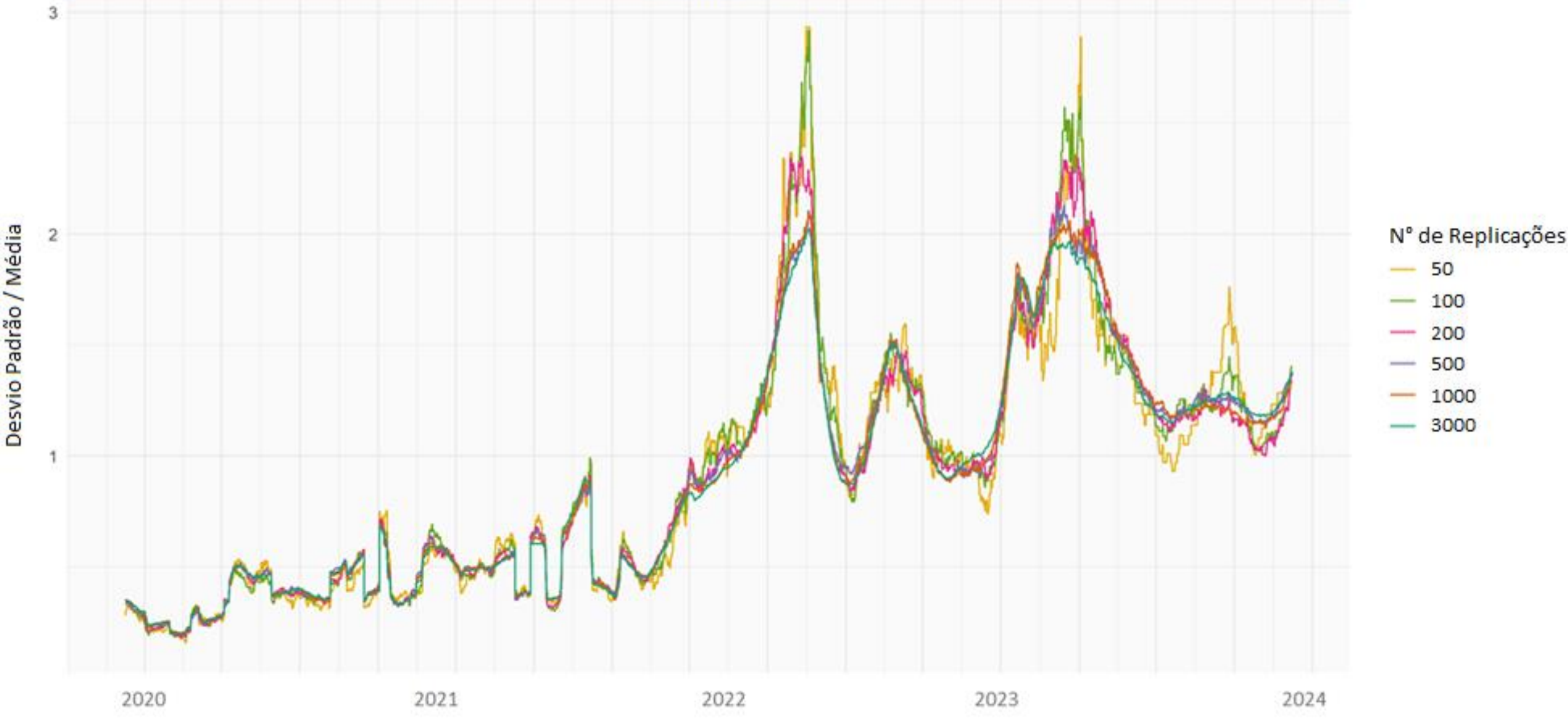




\section{Observação e identificação do problema}

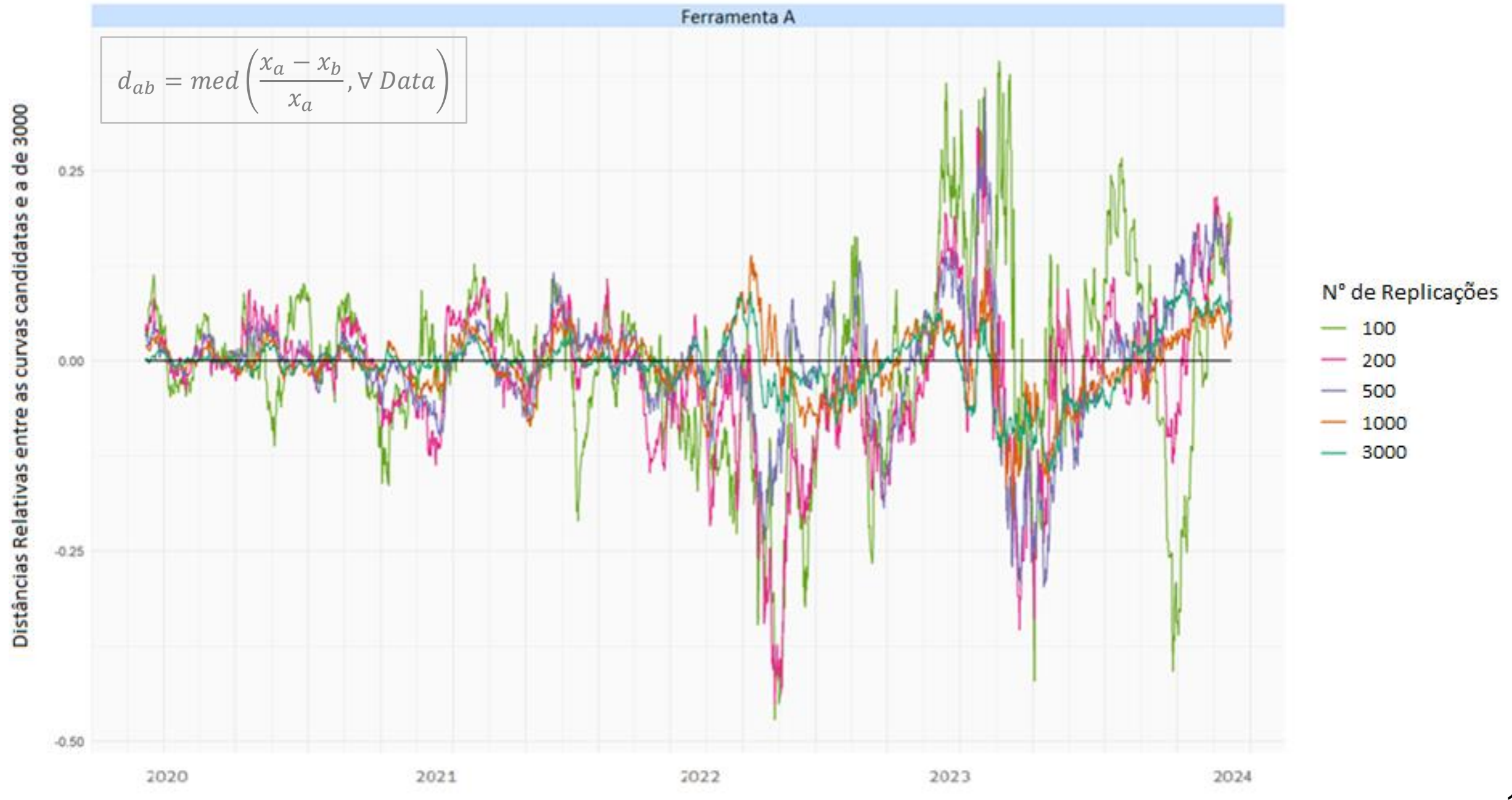




\section{Observação e identificação do problema}

- Média das distâncias relativas para todo o horizonte - representa o quanto os indicadores de cada quantidade de iterações diferem, em média, da curva de 3000 replicações.

\begin{tabular}{c|c|c|c|c|c|c|c|}
\hline № Replicações & Média & P10 & P50 & P90 & $\begin{array}{c}\text { Desvio } \\
\text { padrão }\end{array}$ & $\begin{array}{c}\text { Desvio padrão/ } \\
\text { Média }\end{array}$ & Variância \\
\hline 50 & $-2,3 \%$ & $41,6 \%$ & $-3,1 \%$ & $-16,8 \%$ & $-26,1 \%$ & $-9,4 \%$ & $-20,3 \%$ \\
\hline 100 & $-6,7 \%$ & $24,9 \%$ & $-3,2 \%$ & $-7,7 \%$ & $-18,7 \%$ & $-3,9 \%$ & $-16,9 \%$ \\
\hline 200 & $-3,4 \%$ & $14,5 \%$ & $-2,2 \%$ & $-2,7 \%$ & $-11,4 \%$ & $-2,1 \%$ & $-9,2 \%$ \\
\hline 500 & $-1,0 \%$ & $5,0 \%$ & $-0,9 \%$ & $-0,6 \%$ & $-4,5 \%$ & $-0,7 \%$ & $-1,2 \%$ \\
\hline 1000 & $-3,3 \%$ & $3,2 \%$ & $0,5 \%$ & $-0,7 \%$ & $-6,5 \%$ & $-1,7 \%$ & $-6,9 \%$ \\
\hline
\end{tabular}




\section{Observação e identificação do problema}

- Média das distâncias relativas para todo o horizonte - representa o quanto os indicadores de cada quantidade de iterações diferem, em média, da curva de 3000 replicações.

\begin{tabular}{c|c|c|c|c|c|c|c|}
\hline № Replicações & Média & P10 & P50 & P90 & $\begin{array}{c}\text { Desvio } \\
\text { padrão }\end{array}$ & $\begin{array}{c}\text { Desvio padrão/ } \\
\text { Média }\end{array}$ & Variância \\
\hline 50 & $-2,3 \%$ & $41,6 \%$ & $-3,1 \%$ & $-16,8 \%$ & $-26,1 \%$ & $-9,4 \%$ & $-20,3 \%$ \\
\hline 100 & $-6,7 \%$ & $24,9 \%$ & $-3,2 \%$ & $-7,7 \%$ & $-18,7 \%$ & $-3,9 \%$ & $-16,9 \%$ \\
\hline 200 & $-3,4 \%$ & $14,5 \%$ & $-2,2 \%$ & $-2,7 \%$ & $-11,4 \%$ & $-2,1 \%$ & $-9,2 \%$ \\
\hline 500 & $-1,0 \%$ & $5,0 \%$ & $-0,9 \%$ & $-0,6 \%$ & $-4,5 \%$ & $-0,7 \%$ & $-1,2 \%$ \\
\hline 1000 & $-3,3 \%$ & $3,2 \%$ & $0,5 \%$ & $-0,7 \%$ & $-6,5 \%$ & $-1,7 \%$ & $-6,9 \%$ \\
\hline
\end{tabular}




\section{Observação e identificação do problema}

- Seleção aleatória: amostras de 500 iterações

$$
d_{a b}=\operatorname{med}\left(\frac{x_{a}-x_{b}}{x_{a}}, \forall \text { Data }\right)
$$

\begin{tabular}{c|c|c|c|c|c|c|c|c|c|c|}
$\begin{array}{c}\text { Distâncias } \\
\text { Relativas }\end{array}$ & 1 & 2 & 3 & 4 & 5 & 6 & 7 & 8 & 9 & 10 \\
\cline { 2 - 13 } & 0 & $-0,00108$ & $-0,01281$ & $-0,01007$ & $-0,01546$ & $-0,00560$ & $-0,00480$ & $-0,00194$ & $-0,00377$ & 0,00445 \\
\hline 2 & $-0,00269$ & 0 & $-0,01453$ & $-0,01130$ & $-0,01795$ & $-0,00616$ & $-0,00531$ & $-0,00238$ & $-0,00351$ & 0,00374 \\
\hline 3 & 0,00985 & 0,00977 & 0 & 0,00178 & $-0,00362$ & 0,00544 & 0,00672 & 0,00918 & 0,00759 & 0,01601 \\
\hline 4 & 0,00556 & 0,00574 & $-0,00537$ & 0 & $-0,00710$ & 0,00196 & 0,00279 & 0,00527 & 0,00397 & 0,01253 \\
\hline 5 & 0,01098 & 0,01019 & 0,00018 & 0,00374 & 0 & 0,00641 & 0,00676 & 0,01053 & 0,00818 & 0,01703 \\
\hline 6 & 0,00220 & 0,00320 & $-0,00943$ & $-0,00580$ & $-0,01226$ & 0 & $-0,00011$ & 0,00157 & 0,00065 & 0,00866 \\
\hline 7 & 0,00080 & 0,00191 & $-0,01031$ & $-0,00718$ & $-0,01443$ & $-0,00225$ & 0 & 0,00093 & 0,00009 & 0,00791 \\
\hline 8 & $-0,00228$ & $-0,00111$ & $-0,01383$ & $-0,01056$ & $-0,01626$ & $-0,00657$ & $-0,00496$ & 0 & $-0,00332$ & 0,00469 \\
\hline 9 & $-0,00091$ & 0,00101 & $-0,01217$ & $-0,00864$ & $-0,01554$ & $-0,00421$ & $-0,00261$ & $-0,00012$ & 0 & 0,00611 \\
\hline 10 & $-0,00832$ & $-0,00749$ & $-0,01947$ & $-0,01583$ & $-0,02233$ & $-0,01198$ & $-0,01059$ & $-0,00786$ & $-0,00966$ & 0 \\
\hline
\end{tabular}




\section{Observação e identificação do problema}

- Incertezas causam pouca variabilidade nos cronogramas criados

- Cenários ficam muito similares 


\section{Definição do problema genérico}

\section{Grande Base de Dados}

Cenários pré-determinados

Grande número de replicações

Muitas e complexas etapas de cálculo

Limitações computacionais 


\section{Solução do problema genérico}

\begin{tabular}{|c|c|}
\hline Grande Base de Dados \\
\hline Grande número de replicações pré-determinados & \\
Muitas e complexas etapas de cálculo & Seleção \\
de \\
Cenários
\end{tabular}




\section{Solução do problema genérico}

\begin{tabular}{|c|c|c|}
\hline \multirow{5}{*}{$\begin{array}{l}\text { Seleção } \\
\text { de } \\
\text { Cenários }\end{array}$} & $\Rightarrow$ & Cenários representativos \\
\hline & $\square$ & Mínima perda de confiabilidade \\
\hline & $\longrightarrow$ & Redução do volume de dados \\
\hline & $\longrightarrow$ & Redução do tempo de processamento \\
\hline & & Representação correta das incertezas \\
\hline
\end{tabular}




\section{Proposta de solução}

\section{SELEÇÃO DE CENÁRIOS COM WEIGHTED SET COVERING}

Cenários:

Distância:

Valor de corte:

Arestas:

Critério de cobertura:

$$
S=\left\{s_{1}, s_{2}, \ldots, s_{n}\right\} \text {, onde } s_{d} \in S
$$

$$
f: S \times S \rightarrow \mathbb{R}^{+}
$$

$$
\rho \in \mathbb{R}^{+}
$$

$$
E=\left\{\left(s, s^{\prime}\right) \mid s \in S \wedge s^{\prime} \in S\right\}
$$

$$
C_{s}=\{s\} \cup\left\{s^{\prime} \mid\left(s, s^{\prime}\right) \in S \wedge f\left(s, s^{\prime}\right) \leq \rho\right\}
$$




\section{Proposta de solução}

\section{SELEÇÃO DE CENÁRIOS COM WEIGHTED SET COVERING}

$$
\min z=\sum_{s \in S} f\left(s, s_{d}\right) x_{s}
$$

subject to

$$
\begin{array}{ll}
\sum_{s^{\prime} \in C_{S}} x_{S^{\prime}} \geq 1 & \forall s \in S \\
x_{S} \in\{0,1\} & \forall s \in S
\end{array}
$$




\section{Proposta de solução}

Weighted Set Covering

- Modelo exato

- Heurísticas/Metaheurísticas

Modelo clássico da literatura: K-Medoids 


\section{Resultados Preliminares}

- Matriz de distâncias euclidianas entre as replicações (atributo: mudança na duração)

- Valor de corte: um cenário não pode ser coberto por outro se a distância entre eles for maior que $20 \%$

- Heurística construtiva (first solution)

- Escolhe primeiro os sets com maior número de cenários ainda não cobertos e pára quando obtiver cobertura completa 


\section{Resultados Preliminares}

- Foram selecionados 45 cenários, cuja demanda calculada passou pela mesma análise feita anteriormente

\begin{tabular}{|c|c|c|c|c|c|c|c|c|}
\hline Método & $\begin{array}{c}\text { № } \\
\text { Replicações }\end{array}$ & Média & P10 & P50 & P90 & $\begin{array}{l}\text { Desvio } \\
\text { padrão }\end{array}$ & $\begin{array}{l}\text { Desvio padrão/ } \\
\text { Média }\end{array}$ & Variância \\
\hline Aleatório & 50 & $-2,3 \%$ & $41,6 \%$ & $-3,1 \%$ & $-16,8 \%$ & $-26,1 \%$ & $-9,4 \%$ & $-20,3 \%$ \\
\hline Aleatório & 100 & $-6,7 \%$ & $24,9 \%$ & $-3,2 \%$ & $-7,7 \%$ & $-18,7 \%$ & $-3,9 \%$ & $-16,9 \%$ \\
\hline Aleatório & 200 & $-3,4 \%$ & $14,5 \%$ & $-2,2 \%$ & $-2,7 \%$ & $-11,4 \%$ & $-2,1 \%$ & $-9,2 \%$ \\
\hline Aleatório & 500 & $-1,0 \%$ & $5,0 \%$ & $-0,9 \%$ & $-0,6 \%$ & $-4,5 \%$ & $-0,7 \%$ & $-1,2 \%$ \\
\hline Aleatório & 1000 & $-3,3 \%$ & $3,2 \%$ & $0,5 \%$ & $-0,7 \%$ & $-6,5 \%$ & $-1,7 \%$ & $-6,9 \%$ \\
\hline Set Cover & 45 & $-1,8 \%$ & $0,5 \%$ & $-0,2 \%$ & $3,2 \%$ & $-0,7 \%$ & $-2,3 \%$ & $-0,4 \%$ \\
\hline
\end{tabular}




\section{Resultados Preliminares}

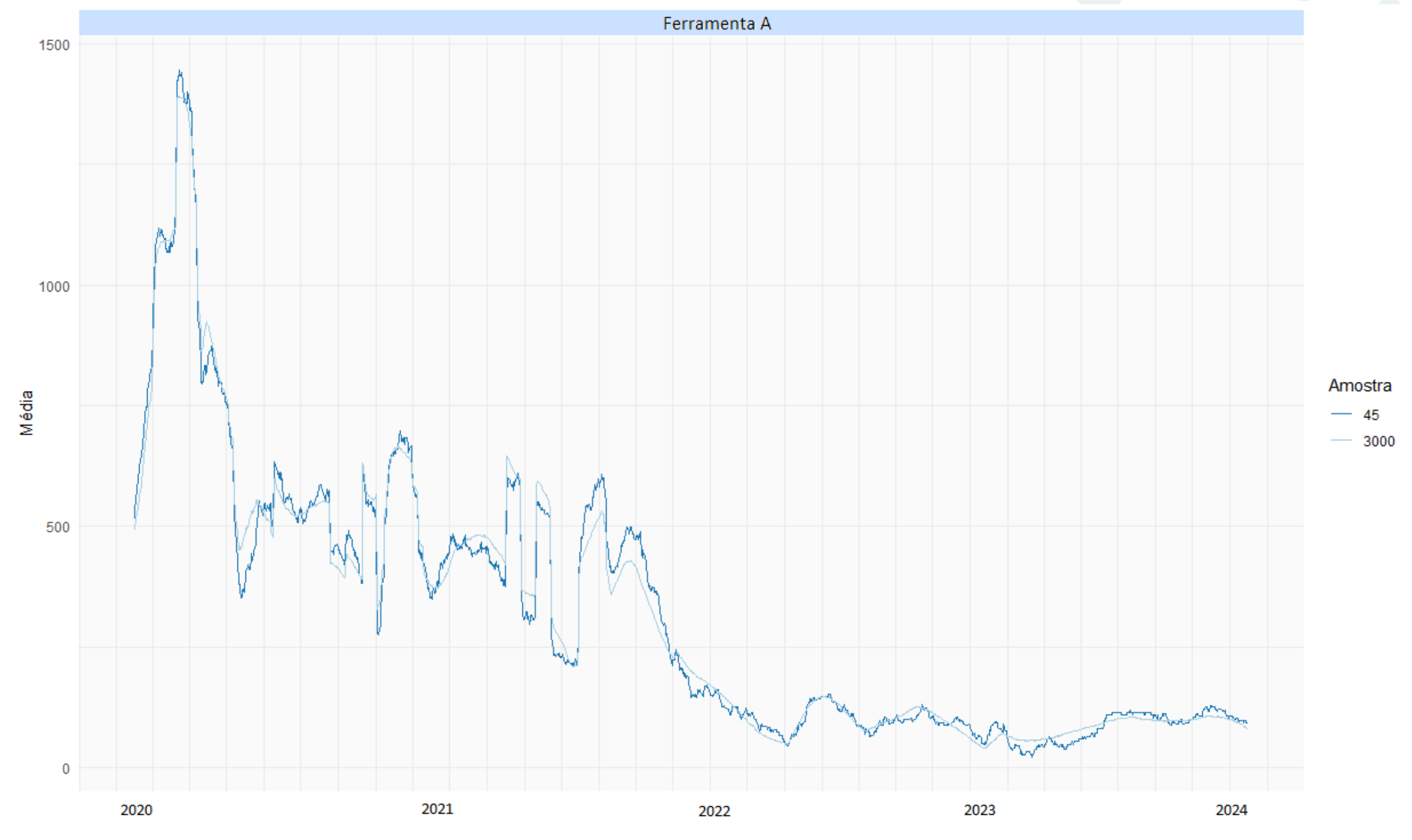




\section{Resultados Preliminares}

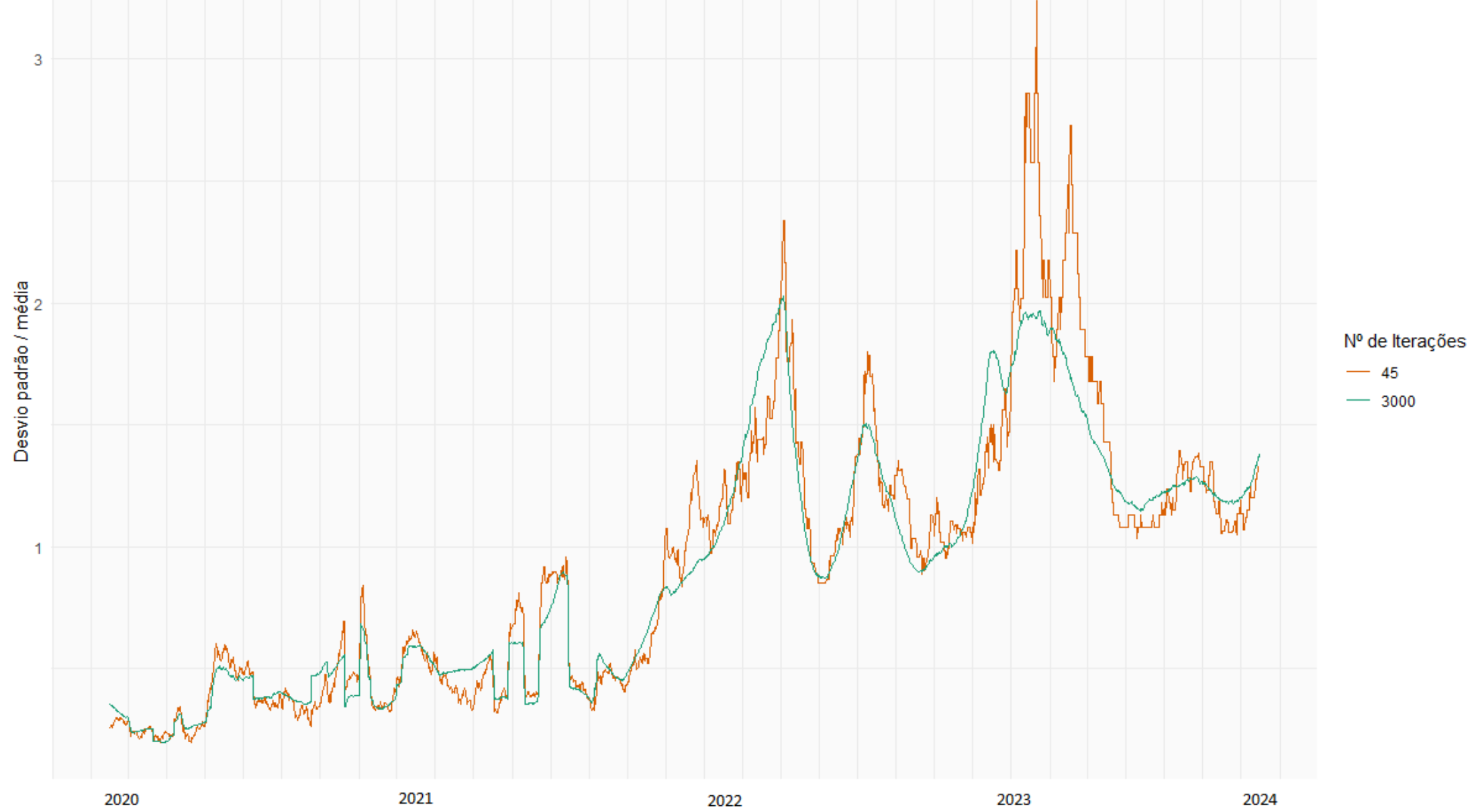




\section{Resultados Preliminares}

Melhorias a serem feitas

- Metaheuristicas e local serch

- Diferentes métodos de cálculo de distância entre cenários

- Análise de sensibilidade de valores de corte 


\section{Obrigada!}

isabellafischer@outlook.com

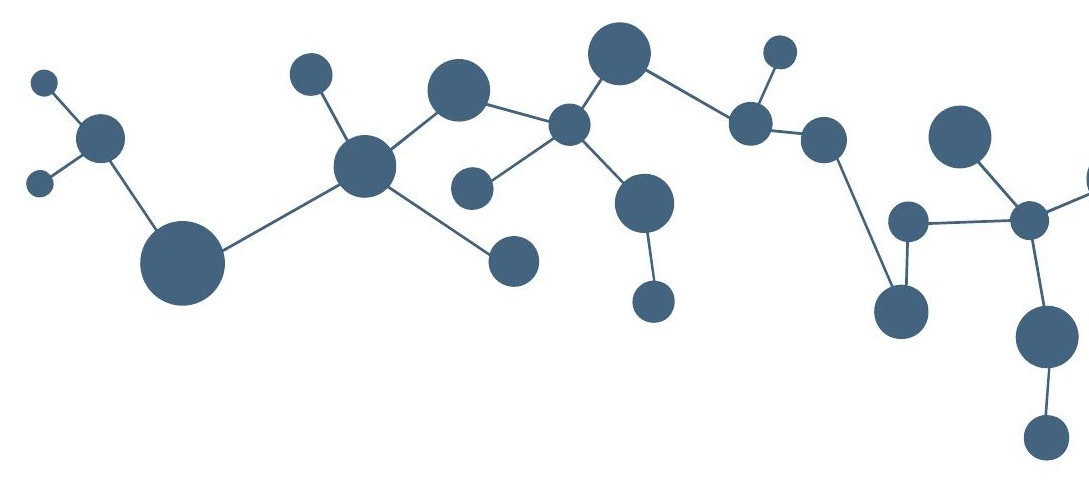

